\title{
Selective Detection of Hydrogen and Hydrogen Containing Gases with Metal Oxide Gas Sensor Operating in Non-Stationary Thermal Regime ${ }^{+}$
}

\author{
Alexey Shaposhnik ${ }^{1}$, Pavel Moskalev ${ }^{1}$ and Alexey Vasiliev ${ }^{2, *}$ \\ 1 Chemistry Chair, Voronezh State agrarian university, Michurin str., 1, Voronezh 394087, Russia; \\ a.v.shaposhnik@gmail.com (A.S.); moskalefff@gmail.com (P.M.) \\ 2 National research center Kurchatov institute, Kurchatov sq., 1, Moscow 123182, Russia \\ * Correspondence: a-a-vasiliev@yandex.ru \\ + Presented at the 8th GOSPEL Workshop. Gas Sensors Based on Semiconducting Metal Oxides: Basic \\ Understanding \& Application Fields, Ferrara, Italy, 20-21 June 2019.
}

Published: 18 June 2019

The main disadvantage of metal oxide (MOX) gas sensors is their insufficient selectivity leading to the dominating application for the quantitative analysis of one-component systems with certain composition, for example, $\mathrm{CH}_{4}$-air or $\mathrm{H}_{2}$-air. The application of non-stationary working regimes (modulation of gas flow, temperature, switch on-off of any chemical converter, etc.) increases the selectivity, because of an increase in the information about the nature of the analite. This improvement of selectivity is possible due to the revealing of the kinetics of the chemisorption of the analite and of the product desorption, the analite interaction with the surface located oxygen anions, etc.

The most know is the application of the non-stationary temperature regime of the sensor operation. The application of temperature modulation in MOX sensors can solve the selectivity problem, only if some important conditions are met. It is necessary (1) to use gas sensitive layer composition leading to different kinetics of the chemisorption and reaction with surface for different gas analites; (2) to find appropriate temperature regime giving extrama in the curve representing electrical resistance as a function of time; (3) to develop the algorithms of the treatment of multi-dimensional data arrays, because the application of non- stationary regime of the sensors operation leads to very significant increase in information volume about gas medium to beanalyzed.

Gas sensing materials based on $\mathrm{Pd}$ decorated $\mathrm{SnO}_{2}$ have at certain temperature regimes characteristic extrema at the curves representing the resistance of the sensing layer as a function of time (and, therefore, temperature, see Figure 1). These extrema are well pronounced at the detection of hydrogen and gases, which can be dehydrogenized at the interaction with the sensing layer (ethanol, acetone, $\mathrm{H}_{2} \mathrm{~S}$, etc.). These curves for hydrogen, ethanol, and $\mathrm{CO}$ (concentration of all gases is of $100 \mathrm{ppm}$ ) are presented in Figure 1. The plots present these functions during two full temperature modulation cycles. 


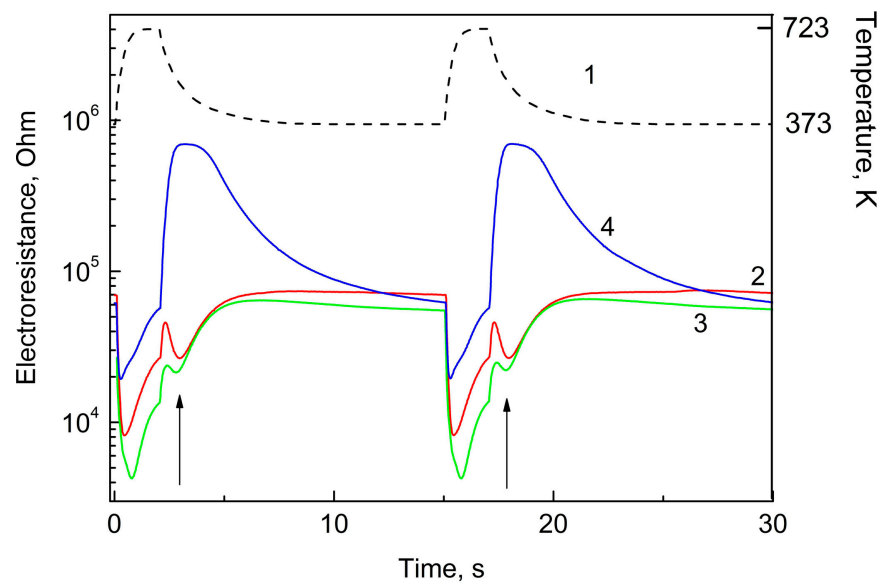

Figure 1. The temperature of the sensor (curve 1) with sensing layer consisting of tin dioxide decorated wiht $\mathrm{Pd}$ and sensing layer resistance at this temperature modulation as a function of time during temperature modulation cycle. (2) 100 ppm of $\mathrm{H}_{2}$, (3) $100 \mathrm{ppm}$ of ethanol, (4) $100 \mathrm{ppm}$ of CO in air.

The extrema on the curves obtained at the detection of hydrogen and ethanol, which is able to release hydrogen at high working temperature of the sensor, are well pronounced. This extremum marked with arrow in the plot is a result of special mechanism of the reaction of the detected gas with the surface. Indeed, the usual mechanism of the interaction includes the reaction with oxygen anions chemisorbed on the surface.

$$
\mathrm{H}_{2}+\mathrm{O}^{-} \rightarrow \mathrm{H}_{2} \mathrm{O}+\bar{\sigma}
$$

Such mechanism is typical of the interaction of other reducing gas (for example, CO, methane) with the surface of the semiconductor. Hydrogen, however, can interact with the surface directly, without the participation of oxygen anions.

$$
K_{2} \rightarrow 2 H^{*}+\overline{Z \theta}
$$

This mechanism can explain the presence of minimum marked with arrow on the plot presenting sensor resistance as a function of time.

During each measurement cycle (Figure 1), we obtained 575 values of sensor resistance giving, respectively, 575 virtual gas sensors. The multi-dimensional data arrays were treated using Principle Component algorithm. Figure 2 shows the possibility to determine both qualitative and quantitative composition of gas mixtures in both one and two component mixtures.

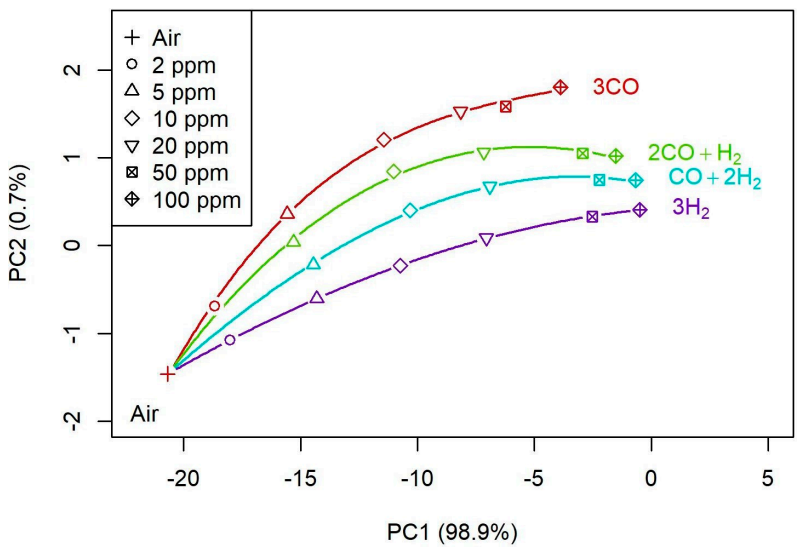

Figure 2. Results of PC analysis of the measurement of 575 virtual sensors. Gas mixtures with the same total concentration and different ratio of $\mathrm{CO}$ and $\mathrm{H}_{2}$ (noted in the plot). 
Funding: This work was funded by RFBR according to the research project No. 18-29-24128

Conflicts of Interest: The authors declare no conflict of interest.

(C) 2019 by the authors. Licensee MDPI, Basel, Switzerland. This article is an open access article distributed under the terms and conditions of the Creative Commons Attribution (CC BY) license (http://creativecommons.org/licenses/by/4.0/). 